\title{
Rotation in string cosmology
}

\author{
Yuri N. Obukhov*, Thoralf Chrobok, and Mike Scherfner ${ }^{\dagger}$ \\ Institut für Theoretische Physik, Technische Universität Berlin, \\ Hardenbergstr. 36, D-10623 Berlin, Germany
}

\begin{abstract}
We describe exact cosmological solutions with rotation and expansion in the low-energy effective string theory. These models are spatially homogeneous (closed Bianchi type IX) and they belong to the family of shear-free metrics which are causal (no closed timelike curves are allowed), admit no parallax effects and do not disturb the isotropy of the background radiation. The dilaton and the axion fields are nontrivial, in general, and we consider both cases with and without the central charge (effective cosmological constant).
\end{abstract}

PACS numbers: PACS no.: 04.20.Cv; 04.20.Jb; 98.80.Cq; 98.80.Hw

\footnotetext{
* On leave from: Department of Theoretical Physics, Moscow State University, 117234 Moscow, Russia

$\dagger$ Institut für Mathematik, Technische Universität Berlin, Str. d. 17. Juni 136, D-10623 Berlin
} 


\section{INTRODUCTION}

The study of cosmological models in the framework of the low-energy effective string theory attracts considerable attention now, see, e.g., [1, 2, 3, 4, 15, 6, 17, 8, 9, 10, 11, 12]. The (super)string theory provides a scheme for the unification of all the physical interactions, including gravity. In the perturbative approach to the full quantum string theory, one derives the effective action for the metric $g$, scalar dilaton field $\phi$ and the 3 -form axion field $H$ which represents the massless field background. The lowest order effective string model describes the Einstein gravity coupled to $\phi$ and $H$.

The string cosmology is of interest, primarily, in connection with the so called pre-big bang phase of the universe's evolution [1, 2], and there are certain expectations that it can improve our understanding of the physics in the range of energies between the string scale and the grand unification (GUT) scale.

Recently the cosmological string models with the nontrivial global rotation have been investigated in [9, 10, 11]. In particular, in [9] the attention was confined to the stationary Gödel type models without expansion, and it was demonstrated that the causal solutions (without closed timelike curves) exist in the first order (in the string tension $\alpha^{\prime}$ ) effective models. These results were subsequently generalized in [10] to the "charged" solutions for the models with scalar and electromagnetic fields included. In an attempt to develop a more realistic cosmological model, the possibility of a rotating and expanding universe was investigated in 11] within the framework of the perturbation theory. A further logical step which can obviously bring a further insight into the rotating cosmologies, should be thus a study of the existence of exact solutions with rotation and expansion. Here we describe such exact models in the low-energy effective string theory, demonstrating that the absence of the closed timelike curves can be combined with the nontrivial expansion and rotation of the universe.

More specifically, in our study we consider the homogeneous Bianchi type IX models with rotation and expansion. The homogeneous Bianchi models in string cosmology were investigated rather extensively in [4, 5, 7, 8, 12]. However, the main efforts were concentrated on the analysis of the evolution of the anisotropy described by the nontrivial shear. For example, the study [8] of the anisotropic Bianchi type has demonstrated the absence of the chaotic behavior of the solutions in an anisotropic string cosmology with shear. In contrast, 
our models are shear-free, and thus we can look into the pure rotational effects in cosmology.

It seems worthwhile to mention that the study of the shear-free models is of interest in itself within the framework of mathematical cosmology. In particular, it is rather difficult to construct physically viable models with nontrivial rotation and expansion in absence of shear: In many cases the traditional sources like an ideal fluid, electromagnetic and scalar fields, etc., simply do not allow for such solutions. At best, it is possible to have either expansion without rotation, or a stationary rotating model without expansion. This constitutes the essence of the so called shear-free conjecture, see an overview in [13]. It is thus highly interesting to discover that the string cosmology (with the standard sources described by the dilaton $\phi$ and the axion $H$ ) does admit exact shear-free solutions with rotation and expansion.

The new solutions are completely causal, in the sense that the closed timelike curves are absent in these rotating models. In this way, we obtain an important extension of the earlier results [9, 10, 11] by demonstrating the existence of an exact (not a perturbation) rotating, expanding, and causal universe in string cosmology.

Another aspect of our results deserves a special attention. Our models describe a spatially closed universe, and we are thus returning to the old problem of the existence of the truly anti-Machian world models. The early model of Ozsváth and Schücking [18] provides an example of such anti-machian cosmology belonging to the Bianchi type IX. Obviously, the model of Ozsváth and Schücking was to a great extent of academic interest only because it described a nonexpanding world. Moreover, it has been subsequently demonstrated that one can reveal a certain compensation mechanism which yields the total angular momentum equal zero for such a closed world [14, 20], thus effectively removing its status of the truly anti-Machian cosmology. Our new solution describes a closed and expanding world with rotation, thus giving a qualitatively new example of an anti-Machian model, cf. also [15]. Furthermore, as far as we can see, there is no compensation scheme similar to [14, 20] for the closed rotating models constructed in the present paper. 


\section{STRING COSMOLOGY}

The lower-energy effective string theory action reads, in the compact notation of exterior calculus,

$$
S=\int e^{-2 \phi}\left(\widehat{R} \widehat{\eta}+4 d \phi \wedge^{\widehat{\star}} d \phi-\frac{1}{2} H \wedge^{\widehat{\star}} H+2 \Lambda \widehat{\eta}\right) .
$$

Here $\widehat{\eta}={ }^{\star} 1$ is the $n$-form of the spacetime volume, $\phi$ is the scalar dilaton field, and the 3 -form field $H=d B$ with the 2 -form potential $B$ is the axion. The quantities with hats refer to the so-called string frame of the 1-form cobasis $\widehat{\vartheta}^{\alpha}$. The effective cosmological constant (or the central charge) is related to the critical dimension of the (super)string theory: $\Lambda=(26-D) / 3 \alpha^{\prime}$ for boson string and $\Lambda=(15-3 D / 2) / 3 \alpha^{\prime}$ for heterotic or superstring. Although this quantity vanishes in critical dimension, it may be nontrivial when a string couples to a conformal theory, for example. Non-rotating solutions in non-critical string cosmologies have been extensively studied in [2, 3, 6]. Like in the latter references, we thus consider $\Lambda$ as an arbitrary parameter. Finally, we will assume that the original higher dimensional theory is reduced to $D=4$ by means of a compactification mechanism.

The field equations read, in components

$$
\begin{aligned}
4 \widehat{D}_{\alpha} \widehat{D}_{\beta} \phi+2 \widehat{R}_{\alpha \beta}-\frac{1}{2} H_{\alpha \mu \nu} H_{\beta}{ }^{\mu \nu} & =0, \\
4 \widehat{D}_{\mu} \widehat{D}^{\mu} \phi-4\left(\widehat{D}_{\mu} \phi\right)\left(\widehat{D}^{\mu} \phi\right)+\widehat{R}-\frac{1}{12} H_{\alpha \mu \nu} H^{\alpha \mu \nu}+2 \Lambda & =0, \\
d^{\widehat{\star}} H-2 d \phi \wedge^{\widehat{\star}} H & =0 .
\end{aligned}
$$

The conformal transformation $\widehat{\vartheta}^{\alpha}=e^{\phi} \vartheta^{\alpha}$ from the string frame to the Einstein frame brings the action (11) to

$$
S=\int\left(R \eta-2 d \phi \wedge^{\star} d \phi-\frac{1}{2} e^{-4 \phi} H \wedge^{\star} H+2 \Lambda e^{2 \phi} \eta\right)
$$

The corresponding field equations read

$$
\begin{aligned}
R_{\alpha \beta}-\frac{1}{2} R g_{\alpha \beta}-\Lambda e^{2 \phi} g_{\alpha \beta} & =T_{\alpha \beta}, \\
D_{\mu} D^{\mu} \phi+\frac{1}{12} e^{-4 \phi} H_{\alpha \mu \nu} H^{\alpha \mu \nu}+\Lambda e^{2 \phi} & =0, \\
d^{\star} H-4 d \phi \wedge^{\star} H & =0 .
\end{aligned}
$$

The right-hand side of Einstein's equations (6) is represented by the energy-momentum tensor of the dilaton and axion fields:

$$
T_{\alpha \beta}=2\left(D_{\alpha} \phi\right)\left(D_{\beta} \phi\right)-\left(D_{\mu} \phi\right)\left(D^{\mu} \phi\right) g_{\alpha \beta}+e^{-4 \phi}\left(\frac{1}{4} H_{\alpha \mu \nu} H_{\beta}{ }^{\mu \nu}-\frac{1}{24} H_{\mu \nu \lambda} H^{\mu \nu \lambda} g_{\alpha \beta}\right) \text {. }
$$


We will study the solutions of the effective string model in the Einstein frame.

\section{METRIC AND THE GEOMETRY OF SPACETIME}

In this paper we continue the investigation of the cosmological models belonging to the wide class of rotating spatially homogeneous metrics [17]

$$
d s^{2}=d t^{2}-2 R n_{a} d x^{a} d t-R^{2} \gamma_{a b} d x^{a} d x^{b}
$$

Hereafter the indices $a, b, c=1,2,3$ label the spatial coordinates, $R=R(t)$ is the scale factor, and

$$
n_{a}=\nu_{A} e_{a}^{A}, \quad \gamma_{a b}=\beta_{A B} e_{a}^{A} e_{b}^{B}
$$

Here $\nu_{A}, \beta_{A B}$ are constant coefficients $(A, B=1,2,3)$, while

$$
e^{A}=e_{a}^{A}(x) d x^{a}
$$

are the invariant 1-forms with respect to the action of a three-parameter group of motion which is admitted by the space-time (10). We assume that this group acts simply-transitively on the spatial ( $t=$ const) hypersurfaces. It is well known that there exist 9 types of such manifolds, distinguished by the Killing vectors $\xi_{A}$ and their commutators $\left[\xi_{A}, \xi_{B}\right]=f_{A B}^{C} \xi_{C}$. The invariant forms (12) solve the Lie equations $\mathcal{L}_{\xi_{B}} e^{A}=0$ for each Bianchi type, so that the models given by (10) are spatially homogeneous.

The models of this class have rather attractive geometrical and physical properties. In particular, we can recall that, in the majority of the earlier cosmological models with rotation, shear manifests itself in the parallax effects and in the distortion of the background radiation which imposes very strong limits on the value of the vorticity [21]. In contrast, one can show [17] that the parallax effects are absent and the microwave background remains isotropic in all shear-free spacetimes (10). As a result, these models satisfy all the known observational tests, including the most important limits from the microwave background radiation analysis [21] which are not applicable to the shear-free geometries. For further de-

tails on the observational tests for such rotating models see 16] Moreover, these models are completely causal (no closed timelike curves are allowed), when the matrix $\beta_{A B}$ is positive definite. 
Previously [14] we have studied Bianchi type IX closed stationary worlds with the spinning fluid as the source. Now, we consider expanding closed worlds belonging to the Bianchi type IX. Denoting the spatial coordinates $x=x^{1}, y=x^{2}, z=x^{3}$, we have explicitly the invariant 1-forms

$$
\begin{aligned}
e^{1} & =\cos y \cos z d x-\sin z d y, \\
e^{2} & =\cos y \sin z d x+\cos z d y, \\
e^{3} & =-\sin y d x+d z,
\end{aligned}
$$

which satisfy the structure equations

$$
d e^{A}=f_{B C}^{A} e^{B} \wedge e^{C}, \quad \text { with } \quad f^{1}{ }_{23}=f^{2}{ }_{31}=f^{3}{ }_{12}=1 .
$$

After these preliminaries, we can write the ansatz for the line element (10)

$$
d s^{2}=g_{\alpha \beta} \vartheta^{\alpha} \vartheta^{\beta}, \quad g_{\alpha \beta}=\operatorname{diag}(1,-1,-1,-1),
$$

in terms of the orthonormal coframe 1-forms $\vartheta^{\alpha}$ :

$$
\vartheta^{\widehat{0}}=d t-R \nu_{A} e^{A}, \vartheta^{\widehat{1}}=R k_{1} e^{1}, \vartheta^{\widehat{2}}=R k_{2} e^{2}, \vartheta^{\widehat{3}}=R k_{3} e^{3}
$$

Here, $k_{1}, k_{2}, k_{3}$ are positive constant parameters. The Greek indices $\alpha, \beta, \ldots=0,1,2,3$ hereafter label the objects with respect to the orthonormal frame; the hats over indices denote the separate frame components of these objects. Using (16) in (15), we find explicitly the $3 \times 3$ matrix:

$$
\beta_{A B}=\left(\begin{array}{ccc}
k_{1}^{2}-\nu_{1}^{2} & -\nu_{1} \nu_{2} & -\nu_{1} \nu_{3} \\
-\nu_{1} \nu_{2} & k_{2}^{2}-\nu_{2}^{2} & -\nu_{2} \nu_{3} \\
-\nu_{1} \nu_{3} & -\nu_{2} \nu_{3} & k_{3}^{2}-\nu_{3}^{2}
\end{array}\right) .
$$

It is straightforward to obtain the kinematical quantities which describe the spacetime geometry. A direct calculation of the vorticity, $\omega_{\mu \nu}=h_{\mu}^{\alpha} h^{\beta}{ }_{\nu} \nabla_{[\alpha} u_{\beta]}$, shear, $\sigma_{\mu \nu}=$ $h^{\alpha}{ }_{\mu} h^{\beta}{ }_{\nu} \nabla_{(\alpha} u_{\beta)}-\frac{1}{3} h_{\mu \nu} \nabla_{\lambda} u^{\lambda}$, and the volume expansion $\theta=\nabla_{\lambda} u^{\lambda}$, yields:

$$
\begin{aligned}
& \sigma_{\mu \nu}=0, \quad a^{\widehat{1}}=\frac{\dot{R} \nu_{1}}{R k_{1}}, \quad a^{\widehat{2}}=\frac{\dot{R} \nu_{2}}{R k_{2}}, \quad a^{\widehat{3}}=\frac{\dot{R} \nu_{3}}{R k_{3}}, \quad \theta=3 \frac{\dot{R}}{R}, \\
& \omega_{\widehat{2} \widehat{ }}=-\frac{\nu_{1}}{2 R k_{2} k_{3}}, \quad \omega_{\widehat{3} \widehat{1}}=-\frac{\nu_{2}}{2 R k_{1} k_{3}}, \quad \omega_{\widehat{1} \widehat{2}}=-\frac{\nu_{3}}{2 R k_{1} k_{2}} .
\end{aligned}
$$

As usually, here $u=\partial_{t}$ is the velocity of a comoving observer (normalized by $u_{\alpha} u^{\alpha}=1$ ) and $h_{\mu \nu}=g_{\mu \nu}-u_{\mu} u_{\nu}$ is the standard projector on his rest 3-space. 


\section{FIELD EQUATIONS AND THEIR EXACT SOLUTIONS}

\section{Axion equation}

The axion field equation (요 is solved by

$$
H=\frac{a_{0}}{R^{3}}\left(\vartheta^{\widehat{1}} \wedge \vartheta^{\widehat{2}} \wedge \vartheta^{\widehat{3}}+\frac{\nu_{1}}{k_{1}} \vartheta^{\widehat{0}} \wedge \vartheta^{\widehat{2}} \wedge \vartheta^{\widehat{3}}+\frac{\nu_{2}}{k_{2}} \vartheta^{\widehat{0}} \wedge \vartheta^{\widehat{3}} \wedge \vartheta^{\widehat{1}}+\frac{\nu_{3}}{k_{3}} \vartheta^{\widehat{0}} \wedge \vartheta^{\widehat{1}} \wedge \vartheta^{\widehat{2}}\right)
$$

Note that one needs also to satisfy the condition $d H=0$ which means the existence of the potential 2-form so that $H=d B$. Here $a_{0}$ is an arbitrary integration constant.

\section{Einstein-dilaton system}

The analysis of the Einstein-dilaton system (66)-(17) ultimately shows that the geometric parameters of the model should satisfy

$$
\nu_{1} \neq 0, \quad \nu_{2}=\nu_{3}=0, \quad k_{2}^{2}=k_{3}^{2}=k_{1}^{2}-\nu_{1}^{2} .
$$

The last equation is particularly important, as it guarantees the absence of closed timelike curves since then the matrix (17) turns out to be explicitly positive definite: $\beta_{A B}=k_{2}^{2} \delta_{A B}$. Making use of (21), one can verify that (6)-(17) reduce to the system of the three equations

$$
\begin{aligned}
\frac{\ddot{R}}{R}+2 \frac{\dot{R}^{2}}{R^{2}}+\frac{k_{1}^{2}}{2 k_{2}^{4} R^{2}}-\frac{k_{1}^{2}}{k_{2}^{2}} \Lambda e^{2 \phi} & =0, \\
-\frac{\ddot{R}}{R}+\frac{\dot{R}^{2}}{R^{2}}+\frac{k_{1}^{2}}{4 k_{2}^{4} R^{2}}-\frac{e^{-4 \phi} a_{0}^{2}}{4 R^{6}}-\dot{\phi}^{2} & =0, \\
\ddot{\phi}+3 \frac{\dot{R}}{R} \dot{\phi}-\frac{e^{-4 \phi} a_{0}^{2}}{2 R^{6}}+\frac{k_{1}^{2}}{k_{2}^{2}} \Lambda e^{2 \phi} & =0 .
\end{aligned}
$$

These three equations are not independent. There is a first integral

$$
3 \frac{\dot{R}^{2}}{R^{2}}+\frac{3 k_{1}^{2}}{4 k_{2}^{4} R^{2}}-\dot{\phi}^{2}-\frac{k_{1}^{2}}{k_{2}^{2}} \Lambda e^{2 \phi}-\frac{e^{-4 \phi} a_{0}^{2}}{4 R^{6}}=0
$$

which provides the consistency of the system (22)-(24). The unknown scale factor $R(t)$ and the dilaton field $\phi$ can be determined from the integration of (25) together with any one of the equations (22)-(24) (the two remaining equations are then satisfied automatically). 


\section{Simple solution}

Before we analyze the Einstein-dilaton system in detail, one can readily find one particular solution:

$$
e^{\phi}=\frac{Q}{R}, \quad R=R_{0}+\frac{k_{1}}{2 k_{2}^{2}} P t, \quad a_{0}=\frac{k_{1}}{k_{2}^{2}} Q^{2} .
$$

Here $Q$ and $P$ are the two arbitrary constants which have to fulfill the condition

$$
P^{2}+1-2 \Lambda Q^{2} k_{2}^{2}=0 .
$$

When $2 \Lambda Q^{2} k_{2}^{2}=1$, the rotating world is stationary, but for $2 \Lambda Q^{2} k_{2}^{2}>1$ the cosmological scale factor monotonously grows with the linear law.

\section{Convenient change of variables}

Let us introduce instead of the scale factor $R(t)$ and the dilaton field $\phi(t)$ the new variables

$$
\psi:=\ln R+\phi, \quad r:=\ln R+\frac{1}{3} \phi .
$$

If we simultaneously change the independent time variable from $t$ to

$$
\tau:=\int^{t} d t e^{\phi}, \quad \text { i.e. } \quad \frac{d}{d \tau}=e^{-\phi} \frac{d}{d t},
$$

then the system of the equations (25) and (23), (24) is re-casted into

$$
\begin{aligned}
2\left(\frac{d \psi}{d \tau}\right)^{2}-6\left(\frac{d r}{d \tau}\right)^{2}+V(\psi) & =0 \\
\frac{d^{2} r}{d \tau^{2}}+\left(\frac{d \psi}{d \tau}\right)^{2} & =0 \\
\frac{d^{2} \psi}{d \tau^{2}}+3 \frac{d r}{d \tau} \frac{d \psi}{d \tau}+\frac{1}{4} \frac{d V}{d \psi} & =0
\end{aligned}
$$

where the potential $V=V(\psi)$ reads

$$
V(\psi)=\frac{a_{0}^{2}}{3} e^{-6 \psi}-\frac{k_{1}^{2}}{k_{2}^{4}} e^{-2 \psi}+\frac{4 k_{1}^{2}}{3 k_{2}^{2}} \Lambda .
$$

The system (301)-(32) has exactly the form of the evolution equations in the so-called "shifted frame" introduced in [6]. The potential (B33) has formally the same structure as in [6], but we have certain corrections of the coefficients in $V$ by the rotation parameters.

In order to understand the behavior of the solutions, we need to analyze the two subcases: (i) the central charge term dominating in $V$ (this takes place for the large positive values of $\psi$ ), (ii) the negligible (or zero) central charge term. 


\section{Dominating cosmological term}

In the case when $\psi$ have large positive values, the potential $V(\psi)$ is dominated by the

central charge (cosmological) term and the potential is approximately $V=\frac{4}{3} \frac{k_{1}^{2}}{k_{2}^{2}} \Lambda$. The solution of (30)-(32) is straightforward and we obtain for the positive $\Lambda$ :

$$
\begin{aligned}
& \psi(\tau)=-\frac{1}{\sqrt{3}} \ln \tanh (A \tau / 2)+\psi_{0}, \\
& r(\tau)=\frac{1}{3} \ln \sinh (A \tau)+r_{0} .
\end{aligned}
$$

Here $\psi_{0}$ and $r_{0}$ are integration constants and

$$
A=\frac{k_{1}}{k_{2}} \sqrt{2 \Lambda}
$$

The original scale factor and the dilaton field then read:

$$
\begin{aligned}
R^{2} & =R_{0}^{2} \sinh (A \tau)(\tanh (A \tau / 2))^{1 / \sqrt{3}} \\
e^{2 \phi} & =\frac{e^{2 \phi_{0}}}{\sinh (A \tau)(\tanh (A \tau / 2))^{\sqrt{3}}} .
\end{aligned}
$$

A solution with $\Lambda<0$ can be obtained with the help of the evident analytical continuation. It is worthwhile to note that, since the constant parameters are always given by (21), the closed timelike curves are absent for all values of the central charge.

The above formulas describe the behavior of the system near the singularity $(R=0)$ when for small moments of $\tau$ the functions $\psi$ and $r$ take very large values. In this regime, the cosmological term is dominating in the potential $V$.

\section{Vanishing cosmological term}

Now let us analyze the case of the critical string cosmology when $\Lambda=0$. It is convenient to take (22) and (25) as the dynamical equations in this case. The equation (22) then can be straightforwardly integrated and yields the first integral:

$$
R^{4}\left(\dot{R}^{2}+\frac{k_{1}^{2}}{4 k_{2}^{4}}\right)=\frac{C_{0}^{2}}{3} .
$$

Here the integration constant is necessarily non-negative and we denoted it by $C_{0}^{2} / 3$ (the coefficient $1 / 3$ is introduced for later convenience). 


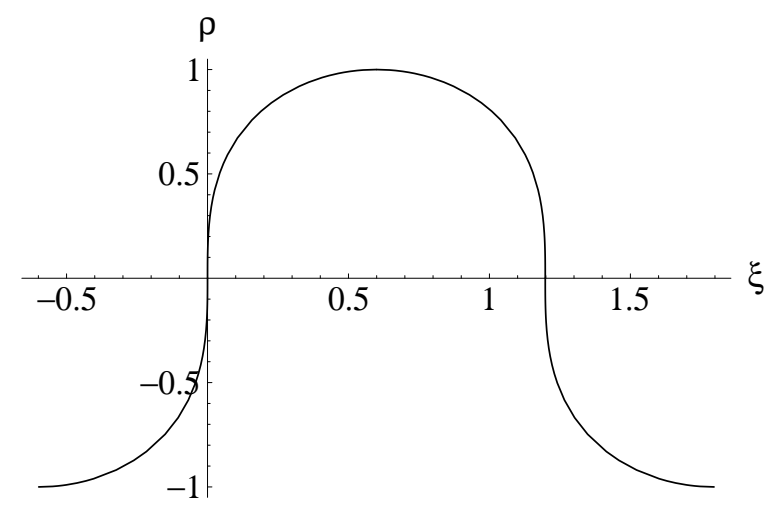

FIG. 1: Scale parameter $\rho=R / R_{0}$ as a function of the cosmological time $\xi=t / t_{0}$ (for $\xi_{0}=0$ ).

Introducing the dimensionless variables, $\rho:=R / R_{0}$ and $\xi:=t / t_{0}$ with

$$
R_{0}^{4}=\left(\frac{2 C_{0} k_{2}^{2}}{\sqrt{3} k_{1}}\right)^{2}, \quad t_{0}^{2}=\frac{3}{C_{0}^{2}}\left(\frac{2 C_{0} k_{2}^{2}}{\sqrt{3} k_{1}}\right)^{3}
$$

we reduce (39) to the quadratures

$$
\int d \rho \frac{\rho^{2}}{\sqrt{1-\rho^{4}}}= \pm \int d \xi
$$

Integrating this, we obtain the solution $\rho(\xi)$ in the implicit form:

$$
E(\arcsin \rho \mid-1)-F(\arcsin \rho \mid-1)= \pm\left(\xi-\xi_{0}\right),
$$

where $F(\varphi \mid m)$ and $E(\varphi \mid m)$ are (first and second kind) elliptic integral special functions. Another (equivalent) representation of the same solution uses the hypergeometric function:

$$
\frac{\rho^{3}}{3}{ }_{2} F_{1}\left(\frac{1}{2}, \frac{3}{4}, \frac{7}{4} ; \rho^{4}\right)= \pm\left(\xi-\xi_{0}\right)
$$

The behavior of the scale factor as a function of time is depicted in Fig. 1.

It is now easy to find the dilaton field by making use of equation (25). Substituting (39) into (25), we integrate the resulting equation to find:

$$
e^{2 \phi}=\frac{1}{4 C_{0}}\left[\exp \left(2 C_{0} \int \frac{d t}{R^{3}}\right)+a_{0}^{2} \exp \left(-2 C_{0} \int \frac{d t}{R^{3}}\right)\right] .
$$

One can do more and obtain the explicit form of the function $e^{2 \phi}(R)$ in terms of the scale factor. Using the above solution, we derive:

$$
e^{2 \phi}=\frac{1}{4 C_{0}}\left[C_{1}\left(\frac{R^{2}}{1+\sqrt{1-\left(R / R_{0}\right)^{4}}}\right)^{ \pm \sqrt{3}}+\frac{a_{0}^{2}}{C_{1}}\left(\frac{R^{2}}{1+\sqrt{1-\left(R / R_{0}\right)^{4}}}\right)^{\mp \sqrt{3}}\right]
$$


Here $C_{1}$ is a new arbitrary integration constant.

Note that although the formulas obtained describe the exact solution for $\Lambda=0$, they also display the approximate behavior of the system when the cosmological term can be neglected as compared to the 2 other terms in the potential $V$.

\section{Exact rotating solution}

The combination of the results of the two previous subsections yields the complete dynamics of the axion-dilaton-graviton system for the nontrivial $\Lambda$. However, besides the above approximate configurations, there always exists an infinite family of the complete exact solutions of (30)-(32) which is parametrized by an arbitrary real papameter. These solutions are obtained when the cosmic rotation parameters satisfy the condition

$$
\frac{k_{1}^{2}}{k_{2}^{8}}=8 a_{0}^{2} \Lambda^{2} .
$$

Then, denoting

$$
e^{2 \psi_{0}}:=\frac{1}{4 k_{2}^{2} \Lambda},
$$

we can introduce the "Hubble function"

$$
H= \pm \frac{A}{3}\left(1-e^{2\left(\psi_{0}-\psi\right)}\right)^{3 / 2}
$$

[with the constant $A$ given by (36)] such that the potential (33) is given by $V(\psi)=6 H^{2}-$

$2(d H / d \psi)^{2}$. The system (30)-(32) is then evidently integrated by setting $d r / d \tau=H$ and $d \psi / d \tau=-d H / d \psi$. As a result, the solution can be given explicitly in the parametric form:

$$
\begin{aligned}
& r(\psi)-r\left(\psi_{0}\right)=-\int_{\psi_{0}}^{\psi} \frac{H(\psi)}{H^{\prime}(\psi)} d \psi, \\
& \tau(\psi)-\tau\left(\psi_{0}\right)=-\int_{\psi_{0}}^{\psi} \frac{1}{H^{\prime}(\psi)} d \psi .
\end{aligned}
$$

Using (48), the integrals are straightforwardly evaluated to give

$$
\begin{aligned}
r(\psi)-r\left(\psi_{0}\right) & =\frac{1}{3}\left(\psi-\psi_{0}\right)-\frac{1}{6}\left(e^{2\left(\psi-\psi_{0}\right)}-1\right) \\
\tau(\psi)-\tau\left(\psi_{0}\right) & =\mp \frac{1}{2 A}\left[e^{\psi-\psi_{0}} \sqrt{e^{2\left(\psi-\psi_{0}\right)}-1}+\ln \left(e^{\psi-\psi_{0}}+\sqrt{e^{2\left(\psi-\psi_{0}\right)}-1}\right)\right] .
\end{aligned}
$$

These results can be directly compared to the non-rotating solution of [ 6$]$ which is recovered in the limit of the vanishing rotation parameter $\nu_{1}=0$. A qualitative new feature, as 
compared to [6], is that the value of the axion integration constant $a_{0}$ is not rigidly fixed by the central charge $\Lambda$ : there always exists a nontrivial $\nu_{1}$ which satisfies (46) for any $a_{0}$ and $\Lambda$.

\section{DISCUSSION AND CONCLUSION}

In this paper, we have studied the problem of universal rotation in string cosmology. We have demonstrated the existence of a number of cosmological models with rotation and expansion which are exact solutions of the low-energy effective string (non-critical, in general) field equations for the metric, dilaton and axion. These solutions are spatially homogeneous, belonging to the type IX of the Bianchi classification. The models under consideration are parallax-free and have an isotropic background radiation, being thus in a good agreement with observations [17]. Moreover, the solutions obtained are completely causal: the absence of closed timelike curves is guaranteed by the positive definiteness of the matrix $\beta_{A B}=k_{2}^{2} \delta_{A B}$ which follows from (21).

The magnitude of the universal rotation for all solutions is given by

$$
\omega^{2}=\frac{1}{2} \omega_{\alpha \beta} \omega^{\alpha \beta}=\frac{\nu_{1}^{2}}{4 R^{2} k_{2}^{4}}=\frac{1}{4 R^{2}}\left(\frac{k_{1}^{2}}{k_{2}^{4}}+\frac{1}{k_{2}^{2}}\right) .
$$

Correspondingly, the vorticity decreases with the growth of the scale factor.

It is worthwhile to mention that, among other solutions, we derive the simple solution (26) with the scale factor increasing linearly in time. The (constant) expansion "velocity" $\dot{R}$ is essentially determined by the rotation parameters, in particular, it becomes faster with an increasing rotation.

Other solutions of the system (22)-(24) turn out to be closely related to the non-rotating solutions of [6] which is based on the possibility to recast the original system into the form (30)-(32) of the so-called "shifted frame" introduced in [6]. The analysis of the system (30)(32) shows that it is sufficient to consider the two cases when $\Lambda$ is, respectively, essentially larger and smaller than the other terms in the "potential" (33). In the case of a dominating central charge (cosmological) term, the approximate behavior of the scale factor and of the dilaton is given by (37), (38) which describe the fields near the cosmological singularity at $R=0$. As we see, the large values of the rotation parameter $\nu_{1}$ yield a more rapid growth of the scale factor, whereas the vorticity quickly decreases in time. 
When the cosmological term vanishes, we derive an exact solution for the system (22)(24). It is given by the equations (42) or (43) and (45). For a large value of rotation constants $\left(k_{1} \gg k_{2}\right)$, we again see that the scale factor increases rapidly in time, whereas the vorticity quickly dilutes during the expansion. Such a behavior is also approximately correct for small values of the cosmological term in (33).

Finally, there always exists an exact solution with cosmic rotation parameters fulfilling the condition (46). It is possible to find a suitable $\nu_{1}$ for any values of the axion constant $a_{0}$ and the cosmological term $\Lambda$. These exact solutions are given by (51), (52) and they represent the direct generalization of the non-rotating solutions discussed earlier in [6].

Summarizing, we find that all the exact solutions which describe the expanding and rotating cosmological models have the same qualitative properties: The large rotation parameters accelerate the expansion of the world, or, in the case of a contracting phase, this decelerates the contraction rate. However, the rotation apparently cannot prevent the occurrence of cosmological singularities, at least in the low energy approximation. The study of higher order corrections to the effective string action may change this conclusion.

The last but not least remark: Since the solutions obtained describe the spatially closed world, their very existence represents the evidence of the possibility of the truly anti-Machian cosmological models. This observation extends the previous results [14, 15, 18] to the case of the more physically realistic closed models with rotation and expansion.

\section{ACKNOWLEDGMENTS}

The authors would like to thank A. Tseytlin for the useful hints and comments about the effective string theory. This work was supported by the Deutsche Forschungsgemeinschaft with the grant 436 RUS 17/70/01.

[1] M. Gasperini and G. Veneziano, Astropart. Phys. 1 (1993) 317.

[2] A.A. Tseytlin, Int. J. Mod. Phys. D1 (1992) 223; A.A. Tseytlin, in: String quantum gravity and physics at the Planck energy scale: Erice Proceedings. Ed. N. Sanchez (River Edge, N.J., World Scientific, 1993), p. 202; A.A. Tseytlin, Class. Quantum Grav. 9 (1992) 979; A.A. Tseytlin and C. Vafa, Nucl. Phys. B372 (1992) 443. 
[3] I. Antoniadis, C. Bachas, J. Ellis, and D.V. Nanopoulos, Nucl. Phys. B328 (1989) 117; M. Mueller, Nucl. Phys. B337 (1990) 37; D.S. Goldwirth and M.J. Perry, Phys. Rev. D49 (1994) 5019.

[4] E.J. Copeland, A. Lahiri, and D. Wands, Phys. Rev. D50 (1994) 4868; E.J. Copeland, A. Lahiri, and D. Wands, Phys. Rev. D51 (1995) 1569; A.P. Billyard, A.A. Coley, and J.E. Lidsey, Phys. Rev. D59 (1999) 123505; M. Gasperini and R. Ricci, Class. Quantum Grav. 12 (1995) 677; G.F.R. Ellis, D.C. Roberts, D. Solomons, and P.K.S. Dunsby, Phys. Rev. D62 (2000) 084004.

[5] J.D. Barrow and K.E. Kunze, Phys. Rev. D55 (1997) 623; J.D. Barrow and K.E. Kunze, Phys. Rev. D56 (1997) 741; K.E. Kunze and R. Durrer, Class. Quantum Grav. 17 (2000) 2597.

[6] R. Easther, K. Maeda, and D. Wands, Phys. Rev. D53 (1996) 4247.

[7] N.A. Batakis, Phys. Lett. B353 (1995) 39; N.A. Batakis, Phys. Lett. B353 (1995) 450; N.A. Batakis and A.A. Kehagias, Nucl. Phys. B449 (1995) 248.

[8] J.D. Barrow and M.P. Dabrowski, Phys. Rev. D57 (1998) 7204.

[9] J.D. Barrow and M.P. Dabrowski, Phys. Rev. D58 (1998) 103502.

[10] P. Kanti and C.E. Vayonakis, Phys. Rev. D60 (1999) 103519.

[11] C.-M. Chen, T. Harko, and M.K. Mak, Phys. Rev. D63 (2001) 104013; C.-M. Chen, T. Harko, W.F. Kao, and M.K. Mak, Nucl. Phys. B636 (2002) 159; C.-M. Chen, T. Harko, W.F. Kao, and M.K. Mak, Rotational perturbations of high density matter in the brane cosmology, 16 pp. hep-th/0208033.

[12] C.-M. Chen, T. Harko, and M.K. Mak, Phys. Rev. D63 (2001) 064002; C.-M. Chen, T. Harko, and M.K. Mak, Phys. Rev. D62 (2000) 124016.

[13] J.M.M. Senovilla, C.F. Sopuerta, and P. Szekeres, Gen. Rel. Grav. 30 (1998) 389.

[14] T. Chrobok, M. Scherfner, and Yu.N. Obukhov, Phys. Rev. D63 (2001) 104014.

[15] Yu.N. Obukhov, T. Chrobok, and M. Scherfner, Phys. Rev. D66 (2002) 043518.

[16] M. Scherfner, T. Chrobok, and M. Shefaat, Eds., Colloquium on Cosmic Rotation (Wissenschaft und Technik Verlag: Berlin, 2000).

[17] Yu.N. Obukhov, in: "Colloquium on Cosmic Rotation" (Berlin, Feb. 1998), Eds. M. Scherfner, T. Chrobok, and M. Shefaat (Wissenschaft und Technik Verlag: Berlin, 2000) 23-96; E-print archive: astro-ph/0008106.

[18] I. Ozsváth and E. Schücking, Nature 193 (1962) 1168; I. Ozsváth and E. Schücking, in: Recent 
developments in general relativity (Pergamon: Oxford, 1962) 339; I. Ozsváth and E. Schücking, Ann. Phys. 55 (1969) 166. I. Ozsváth and E.L. Schücking, J. Geom. Phys. 24 (1998) 303; I. Ozsváth, Class. Quantum Grav. 14 (1997) A291.

[19] Ø. Grøn and H.H. Soleng, J. Math. Phys. 29 (1988) 1514; Erratum, J. Math. Phys. 29 (1988) 2320 .

[20] D.H. King, A closed universe cannot rotate, in: Einstein Studies, vol. 6: "Mach's principle: From Newton's bucket to quantum gravity", Eds. J.B. Barbour and H. Pfister (Birkhäuser: Boston, 1995) 237; D.H. King, Phys. Rev. D44 (1991) 2356.

[21] S.W. Hawking, Mon. Not. Roy. Astr. Soc. 142 (1969) 129; C.B. Collins and S.W. Hawking, Mon. Not. Roy. Astr. Soc. 162 (1973) 307; J.D. Barrow, R. Juszkiewicz, and D. Sonoda, Mon. Not. Roy. Astr. Soc. 213 (1985) 917; E. Bunn, P. Ferreira, and J. Silk, Phys. Rev. Lett. 77 (1996) 2883; A. Kogut, G. Hinshaw, and A. Banday, Phys. Rev. D55 (1997) 1901. 